4. Tan CS, Cheong KX, Lim LW, Li KZ. Topographic variation of choroidal and retinal thicknesses at the macula in healthy adults. $\mathrm{Br}$ J Ophthalmol. 2013;98:339-44.
5. Tan CS, Cheong KX. Macular choroidal thicknesses in healthy adults-relationship with ocular and demographic factors. Invest Ophthalmol Vis Sci. 2014;55:6452-8.

\title{
Comment on: Difference of uveal parameters between the acute primary angle closure eyes and the fellow eyes
}

\author{
Xingyi $\mathrm{Li}^{1} \cdot$ Wei Wang ${ }^{1} \cdot$ Xiulan Zhang $\mathbb{1}^{1}$
}

Received: 15 June 2018 / Accepted: 5 July 2018 / Published online: 31 July 2018

(c) The Royal College of Ophthalmologists 2018

We read with interest the comment by Cheong et al. [1].

As the comment mentioned, in our study, the diurnal variations was an insurmountable confounding factor to the thickness of choroid and other parts of uvea. As we know, APAC attack usually happened at night, which was speculated be associated with dilating pupil as a result of dim lighting [2]. The attacks in our study were almost in the same situation. The examinations performing at the same timepoint was the most simple and effective way to reduce the confounding effect. Meanwhile, the previous studies about the diurnal variations of choroidal thickness (CT) found that the maximum at 0300 hours, and the minimum at 1800 hours, which meant the CT gradually expand after sunset until midnight [3]. We speculate the trend of choroid expanding might play a role in the angle closure.

Second, our research team has recently published several articles about the topographic variation of the choroid. We found that POAG/PACD eyes had a thicker anterior choroid than the controls. However, there was no significant difference in the posterior choroidal thickness among the groups. The anterior choroid might play a role in the pathogenesis of glaucoma [4]. Meanwhile we found that Valsalva manoeuvre did not affect the posterior choroid, but it did cause thickening of the anterior choroid and the ciliary body, both of which led to a larger anterior placement of the ciliary body and a narrowed anterior chamber. The anterior (but not the posterior) choroid could be related to IOP

Xiulan Zhang

zhangx12@mail.sysu.edu.cn

1 State Key Laboratory of Ophthalmology, Zhongshan Ophthalmic Center, Sun Yat-Sen University, Guangzhou 510060, China elevation and a narrowed anterior chamber in primary angle closure diseases $[5,6]$. The relationship between the anterior choroid and the blood pressure need further investigation.

Finally, we thank Cheong et al. for the kind comment.

Funding: This research was supported by the National Natural Science Foundation of China (81670847, 81600728). The Cultivation Projects for Young Teaching Staff at Sun Yat-sen University (17ykpy75).

\section{Compliance with ethical standards}

Conflict of interest The authors declare that they have no conflict of interest.

\section{References}

1. Cheong XK, Tan C. Comment on: Difference of uveal parameters between the acute primary angle closure eyes and the fellow eyes. Eye. 2018.

2. Pokhrel PK, Loftus SA. Ocular emergencies. Am Fam Physician. 2007;76:829-36.

3. Usui S, Ikuno Y, Akiba M, et al. Circadian changes in subfoveal choroidal thickness and the relationship with circulatory factors in healthy subjects. Invest Ophthalmol Vis Sci. 2012;53:2300-7.

4. Gao K, Li F, Li Y, et al. Anterior choroidal thickness increased in primary open-angle glaucoma and primary angle-closure disease eyes evidenced by ultrasound biomicroscopy and SS-OCT. Invest Ophthalmol Vis Sci. 2018;59:1270-7.

5. Li X, Wang W, Chen S, et al. Effects of valsalva maneuver on anterior chamber parameters and choroidal thickness in healthy Chinese: an AS-OCT and SS-OCT study. Invest Ophthalmol Vis Sci. 2016;57:T189-95.

6. Li F, Gao K, Li X, et al. Anterior but not posterior choroid changed before and during Valsalva manoeuvre in healthy Chinese: a UBM and SS-OCT study. Br J Ophthalmol. 2017;101:1714-19. 